\title{
VOLTAGE MONITORING INSTRUMENT WITH FAST-TRANSIENTS CAPTURE CAPABILITY
}

\author{
M. Gracida-Aguirre ${ }^{1} \boldsymbol{\&}$ H. Calleja ${ }^{2}$. \\ ${ }^{1}$ Instituto Tecnológico de Cerro Azul, Carretera a Tuxpan, km 60, Cerro Azul, Veracruz, 92510, México, m_gracida@yahoo.com \\ ${ }^{2}$ Cenidet - Departamento de Electrónica, P.O. Box 5-164 Cuernavaca Morelos, 62050, México, hcalleja@cenidet.edu.mx \\ Received December $5^{\text {th }} 2000$ and accepted September $12^{\text {th }} 2002$
}

\section{ABSTRACT}

A monitoring instrument aimed at recording the voltage levels at residential installations is described. Its main feature is the capability ot recording short transients, lasting less than one cycle, while requiring a rather modest amount of memory for long monitoring periods. The instrument uses synchronous sampling of the voltage waveform, and its response to outages has been optimized.

\section{RESUMEN}

Se describe un instrumento de medición enfocado a registrar los niveles de tensión en instalaciones eléctricas residenciales. Su característica principal es la capacidad para registrar transitorios de corta duración, con duración menor a un ciclo de línea; al mismo tiempo, es capaz de almacenar periodos largos de medición utilizando una cantidad modesta de memoria. El instrumento utiliza el muestreo sincrónico de la forma de onda de tensión, y su respuesta a los cortes de energía se ha optimizado.

KEYWORDS: Power metering, Sampling instrument, Power quality.

\section{INTRODUCTION}

Voltage level is an important parameter of power quality [1 - 2]. Utilities usually monitor this parameter at substations and selected points of the distribution grid, or in large industrial facilities, where the cost of complex monitoring equipment is justified [3 - 4]. At residential level, however, there is little information available due, in part, to the high cost of the monitoring devices.

Most of the monitoring devices used by utilities keep track of the voltage on a sequential basis, that is, the voltage averaged during a fixed integrating period is obtained and the result stored in a memory. The average for the next period is stored in the next memory location, and so on. In order to capture fast voltage fluctuations, such as the sag produced by a motor starting, the integrating period must be very short. This, in turn, produces a very long memory record, due to the requirements set by the standards, as a recording device should be capable of keeping track of the monitored variables for a minimum of 35 days.

Furthermore, the voltage level averaged is the RMS value which calculation, by itself, involves an integration. Thus, a double integration is performed: the first to calculate the RMS value and the second to obtain an average over the 
integrating period. As a consequence, short transients, that might cause the malfunction of sensitive equipment, are not recorded.

The monitoring instrument described in this paper offers both low complexity and cost. It is capable of recording voltage levels, including transients lasting less than one line cycle, for long monitoring periods. Since it has been found out that most of the brownouts and outages are rather short, lasting just a few seconds [5], the transient response of the instrument has been optimized, both for the detection of outages and for sampling recovery upon signal reconnection.

\section{DESIGN OBJETIVES}

The monitoring instrument should meet the following design requirements:

- It is to be connected to the electric grid, with a nominal voltage $V_{N}=120$ Volts.

- It should be capable of storing the measured information of, at least, 35 days. The information should be retained even if the instrument is unpowered.

- It should be fast enough to capture transients lasting less than one cycle, and to provide a quick recovery after power interruptions.

- It should provide suitable means to retrieve the recorded data.

- Its construction should be simple and low-cost.

\section{OPERATING PRINCIPLE}

The instrument is basically a data-logger, built around an 8-bit microcontroller. Its main feature is that the information of the voltage waveform is stored as a histogram, in such a way that the recording memory is organized as a set of counters. Each counter is associated with an angle and a voltage level, corresponding to a point in a plane. The value of a counter represents the number of times that the voltage waveform crosses this particular point during the monitoring period. As an example, consider the point $X=(\pi / 2,120 \sqrt{2})$. Under nominal operating conditions, a monitoring device keeping track of this point will register 60 crossings of the waveform during one second. Clearly, this requires that the waveform be sampled on a synchronous basis.

As many points as desired can be defined, depending on the time and amplitude resolutions selected, and each point is associated with a counter. However, in order to reduce the length of the memory used, the waveform is recorded only when it is within the boundaries defined by:

$$
\begin{aligned}
& V_{U}=1.2 V_{N} \\
& V_{L}=0.7 V_{N}
\end{aligned}
$$

for the upper and lower boundary respectivery, as shown in figure 1. As it can be seen, they are not symmetrical around the nominal value since lower levels have a higher probability to occur.

3.1 Time and amplitude resolution

As a starting point, 32 samples per cycle are chosen, as shown in figure 2 for the positive half-cycle. For this number of samples a time resolution of $520.8 \mu \mathrm{sec}$. at $60 \mathrm{~Hz}$ is obtained. 


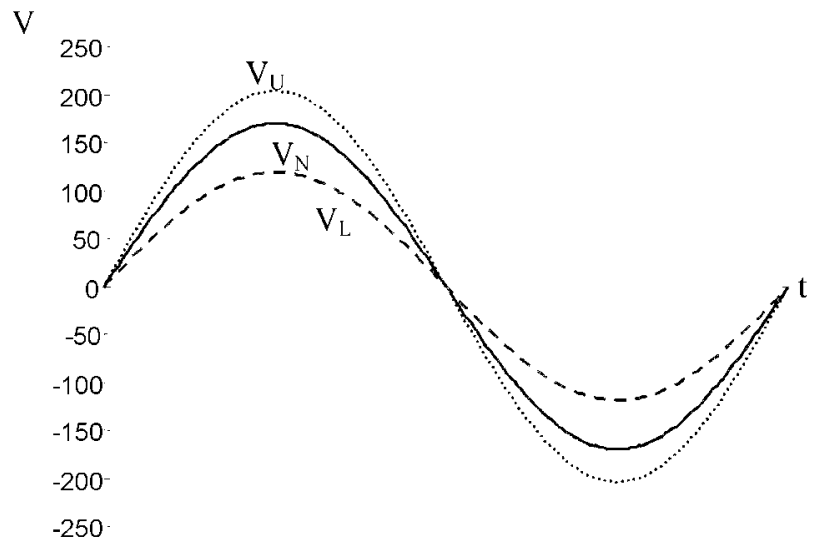

Figure 1. Upper and lower boundaries of the monitoring window.

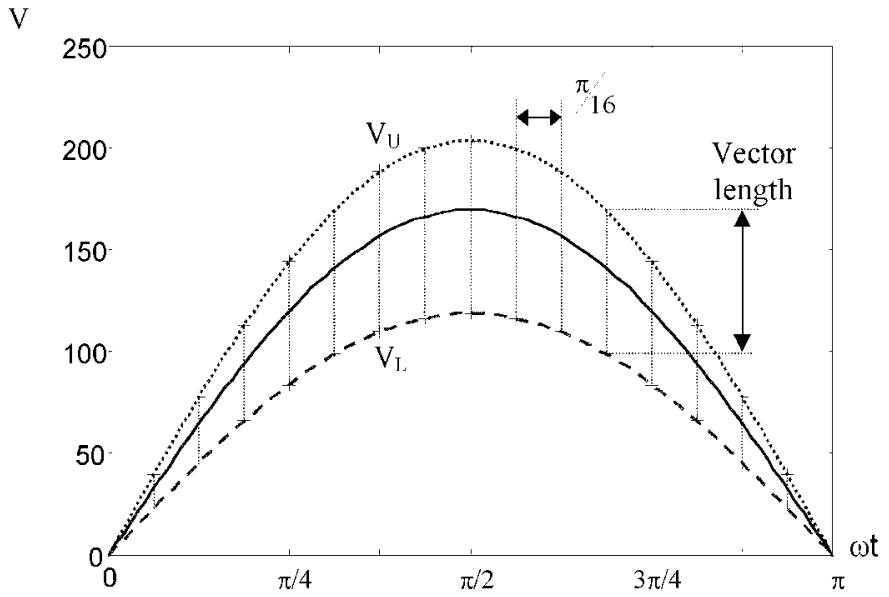

Figure 2. Sampling points for the positive half cycle.

The amplitude resolution is a function of the number of bits $n$ of the analog to digital converter used to digitalize the voltage waveform. The input range of the instrument must cover the full voltage swing of $\mathrm{V}_{\mathrm{u}}$, and the resolution can be expressed as:

$$
V_{R}=\frac{1.2 \sqrt{2} 120 \mathrm{~V}}{2^{n}}
$$

For $n=10$, the resolution obtained is $V_{R}=0.397$ volts

\subsection{Memory length}

The memory amount required for storage is directly related to the resolution. The memory is organized as a set of 32 vectors, each one containing the counters associated with a given sample angle. In turn, the length of each vector is a function of the amplitude resolution and the distance between the boundaries. The length of the $j$ 'th vector is given by: 


$$
\begin{gathered}
M_{j}=\frac{\sqrt{2}}{V_{R}}\left(V_{U}-V_{L}\right) \sin \left(j \frac{\pi}{16}\right) ; j \neq 16,32 \\
M_{j}=1 ; j=16,32
\end{gathered}
$$

In equation (4) both $V_{u}$ and $V_{L}$ are RMS values. Equation (5) covers the case of the samples taken at the zero crossings. The length of the vectors is listed in table I; adding the number of elements in all vectors, it was found out that 4342 counters are needed.

Table I. Length of vectors.

\begin{tabular}{|l|c|c|c|c|c|c|c|c|c|}
\hline \multirow{2}{*}{ Vector } & 0 & 1,15 & 2,14 & 3,13 & 4,12 & 5,11 & 6,10 & 7,9 & 8 \\
\cline { 2 - 10 } & 16 & 17,31 & 18,30 & 19,29 & 20,28 & 21,27 & 22,26 & 23,25 & 24 \\
\hline Length & 1 & 42 & 82 & 119 & 151 & 178 & 197 & 209 & 214 \\
\hline
\end{tabular}

The size of the memory needed to store the vectors is also a function of the monitoring period. There are 5,184,000 cycles/day; if a minimum period of 35 days is desired, then the number of cycles will be:

$$
N_{C}=181.44 \times 10^{6} \frac{\text { cycles }}{\text { period }}
$$

In order to represent $\mathrm{N}_{\mathrm{c}}$, a width of 28 bits is required. In a microcomputer the width of the counters should be multiple of 8 . Thus, each individual counter will be 32 bits wide. The total memory needed to store the vectors is then 17368 bytes.

Since there is always the possibility for the waveforms to be outside the monitoring window, two additional counters per sample point are included. One of them is used to indicate that the waveform is above the window, while the other one indicates that the waveform is below, although this does not necessarily means that an outage has occurred.

\subsection{Address calculation}

For any sampling angle, the particular address $A$, of the $j$ 'th counter is a function of the analog-to-digital conversion result. Since the output of the converter is a binary number $N_{\text {, }}$ the address is calculated with:

$$
A_{j}=A_{B}+4\left(N_{j}-N_{L}\right)
$$

where $N_{\iota}$ is a binary number corresponding to the lower limit of the monitoring window, and $A_{B}$ is the starting address of the vector associated with the sampling angle.

\section{HARDWARE DESCRIPTION}

The block diagram of the monitoring instrument is shown in figure 3. It is based on a general-purpose, low-power 8 bit microcontroller, and includes the following:

- $\quad$ Non volatile memory

The memory is used to store the counters and the data related to the occurrence of outages.

- $\quad$ Real time clock 
The clock is used to keep track of the time of occurrence and duration of the outages; all time tags are obtained from this clock. The use of the line frequency for timing purposes is avoided since it is not tightly regulated.

- A serial communications channel.

This is a standard RS232, used to program the clock and retrieve the recorded information.

- A sampling section.

This includes two parts: a synchronization module and a 10-bit analog to digital converter.

- A battery-backed power supply

This feature is necessary because the instrument must remain operational during power outages.

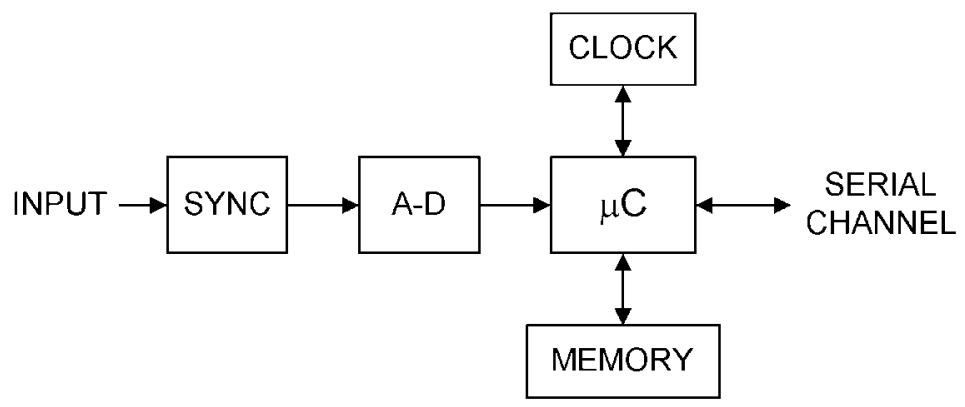

Figure 3. Monitor block diagram.

\subsection{Synchronization module}

The typical architecture of data-loggers has already been described elsewhere [6]; thus, only the synchronization module will be described in detail. The module is shown in figure 4; it is based on an AC voltage detector and a digital $\mathrm{PL}$; there are, however, several additions to the usual construction of a synchronous sampling module: switch $\mathrm{S}$ between the phase detector and the filter, and the reset circuit labeled RST.

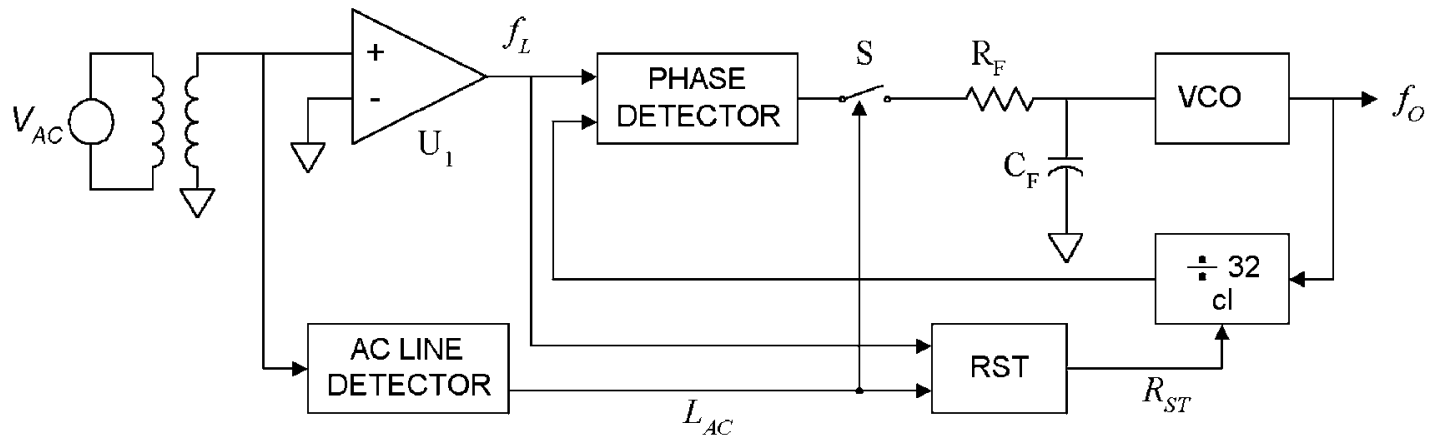

Figure 4. Synchronization module

The voltage waveform is scaled down with a transformer; since all the measurements are related to its secondary winding, it is not necessary to compensate the phase shift between windings. The secondary winding is connected to an AC line detector, specifically designed to have a fast response [7]. Its output, labeled as $L_{\text {ac, }}$ is used to signal the microcontroller that an outage has occurred, and as an input to the reset circuit RST.

The secondary winding is also connected to comparator U1, which provides a square wave labeled $f_{L}$, in phase with the voltage waveform. This signal is used for two purposes: First, it is fed to the microcontroller, which uses it to synchronize the positions of the samples within a cycle. Second, it is also fed to the reset circuit in the sampling module 
The PLL is configured as a frequency multiplier, with a divide-by-32 counter in the feedback loop. When the input signal to a PLL is disconnected, its output frequency usually drifts to the lower limit; when the input signal is reapplied, a transient is generated until the PLL becomes locked again. Clearly, during this transient no reliable sampling can be performed.

The length of the reconnection transient is basically a function of the tuning of the loop and the frequency difference between $f_{o}$ and the incoming frequency. The function of the AC line detector and RST is to reach the locked state as fast as possible, providing accurate sampling of the waveform upon signal reconnection. It prevents the VCO from drifting, keeping $f_{0}$ at the value it had before the signal disconnection. Once the PLL is locked, these circuits have no further effect on the performance of the module.

Under normal operation, the output of the reset block, will be inactive, switch S will be closed and the PLL will be locked to the main frequency. When an outage occurs, the output of the AC line detector goes low; switch $S$ is opened and the capacitor $C_{F}$ in the low-pass filter retains the charge it had at the instance prior to the outage. During the length of the outage, the capacitor will slowly discharge, with a rate dictated by the magnitudes of the leakage currents, and fo will drift at the same rate.

Figure 5 shows the timing sequence for signal reconnection. The AC line is energized again at instant to; after a short delay, $L_{x}$ Switches to the high state at instant $t_{1}$; a short pulse is produced in line RST at the next negative-edge in $f_{t}$ (instant $\mathrm{t}_{2}$ ) resetting the counter. If the outage was short, $\mathrm{f}_{\mathrm{o}}$ will be very close to the value it had prior to the outage but, most likely, will have a different phase. Resetting the counter forces the feedback signal to the phase detector to be in phase with signal $f_{\mathrm{L}}$. The pull-in time of the PL is shortened, providing an almost transient-free operation of the circuit upon signal reconnection [8].

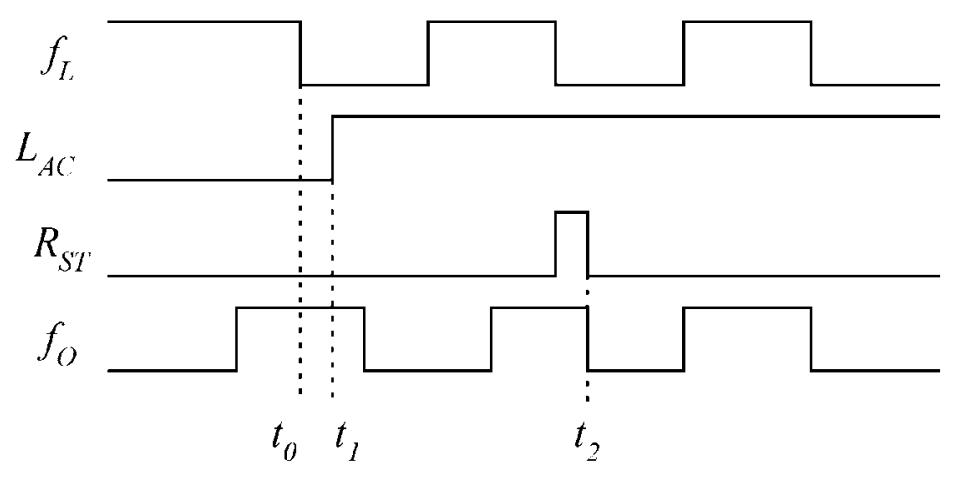

Figure 5. Timing sequence for signal reconnection.

\section{OPERATION}

When powered for the first time, the real-time clock must be initialized. Next, all the vectors are cleared and the microprocessor waits for a positive zero-crossing signal. Sampling starts when this crossing is detected, with a time-tag header recorded in memory. Each individual sample is compared with the limits to ensure that it falls within the monitoring window; if it does, the counter corresponding to the value of the sample is incremented. This procedure continues until a download is requested, or an outage occurs.

When a download request from an external device is requested, typically from portable PC, the microprocessor dumps through the serial channel the content of the full memory record. If the transfer is successfully completed, the memory is cleared, a new time-tag is recorded, and a new measuring period is started.

The outages are detected through the line monitor, with its output switching from high to low. The microprocessor stops sampling the voltage waveform and a time-marked is recorded. The microprocessor then waits for line $L_{c}$ to become active again; when this happens, a new time-marker is recorded. The length of the outage is the difference between the two time-markers. Sampling is started with the first positive zero-crossing following the activation of $\mathrm{L}_{\mathrm{i} .}$. 


\section{RESULTS}

A prototype was built, using a low power version of the 8051 microcontroller. The tests performed included the sampling mechanism and the response to power outages. The sampling behavior is shown in figure 6 , where the samples taken are compared with the actual voltage waveform applied to the instrument.

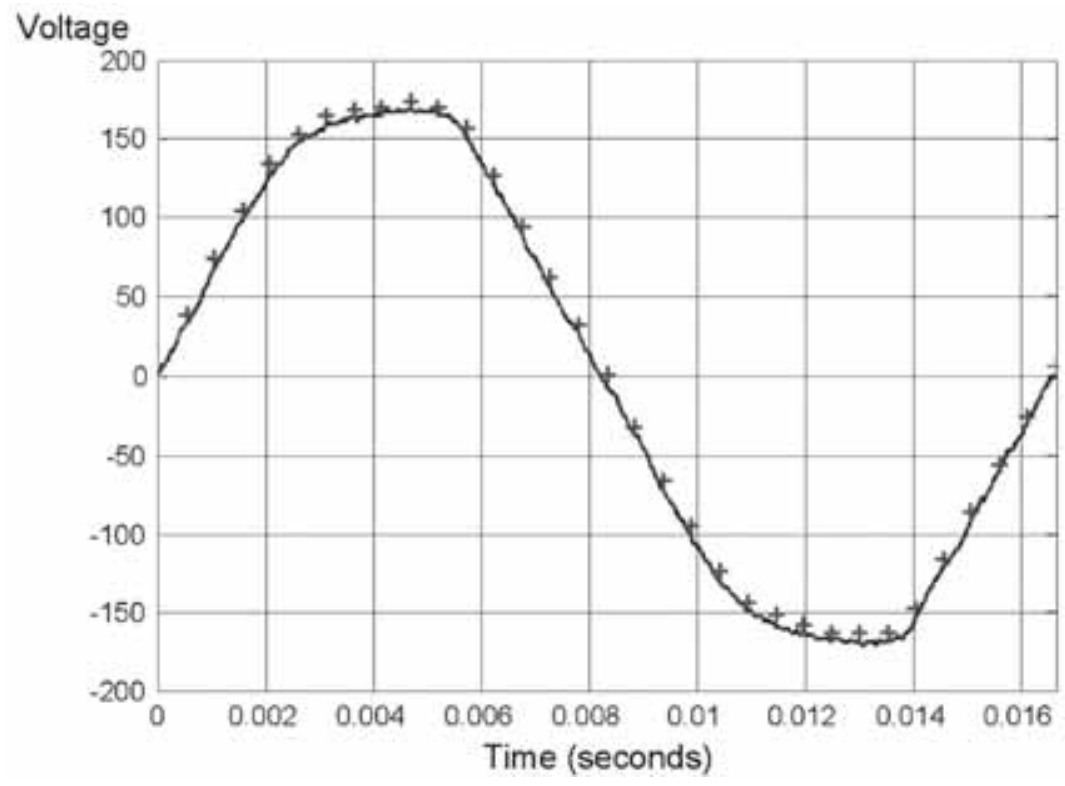

Figure 6. Sampling of a single cycle.

The response of the synchronization circuit is shown in figure 7; where the upper trace corresponds to line $\mathrm{L}_{\mathrm{a}}$, and the lower trace is the voltage at the input of the VCO; as can be seen, the transients due to signal disconnection are practically eliminated.

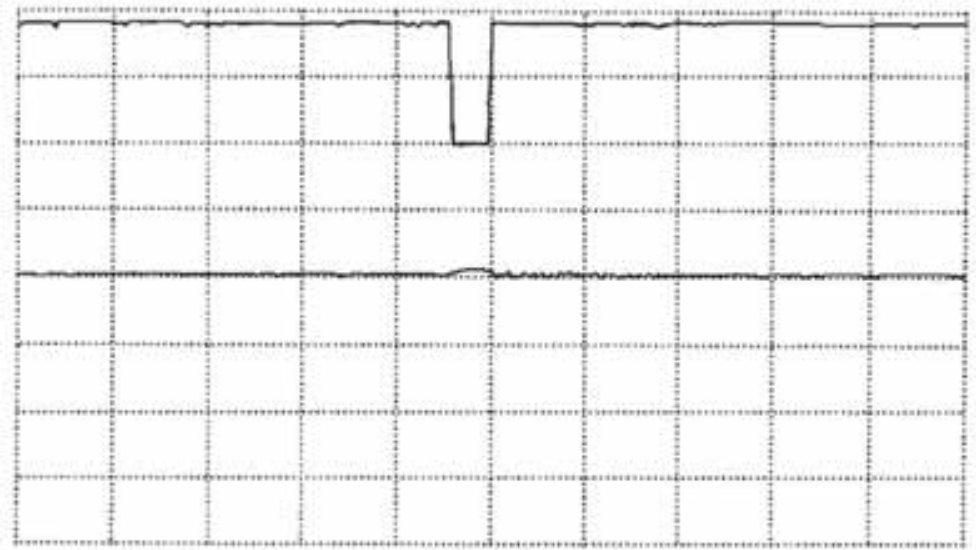

Figure 7. Response of the synchronization circuit. Vertical scale $=1 \mathrm{~V} /$ div; horizontal scale $=1 / \mathrm{sec} /$ div.

Finally, figure 8 shows the effect of the reset circuit; the upper trace is the output of comparator U1, while the lower trace is the output of the counter in the feedback loop of the PLL. Prior to signal reconnection, the feedback signal is almost $180^{\circ}$ out of phase, and is quickly forced to be in phase, thus drastically shortening the pull-in time. 


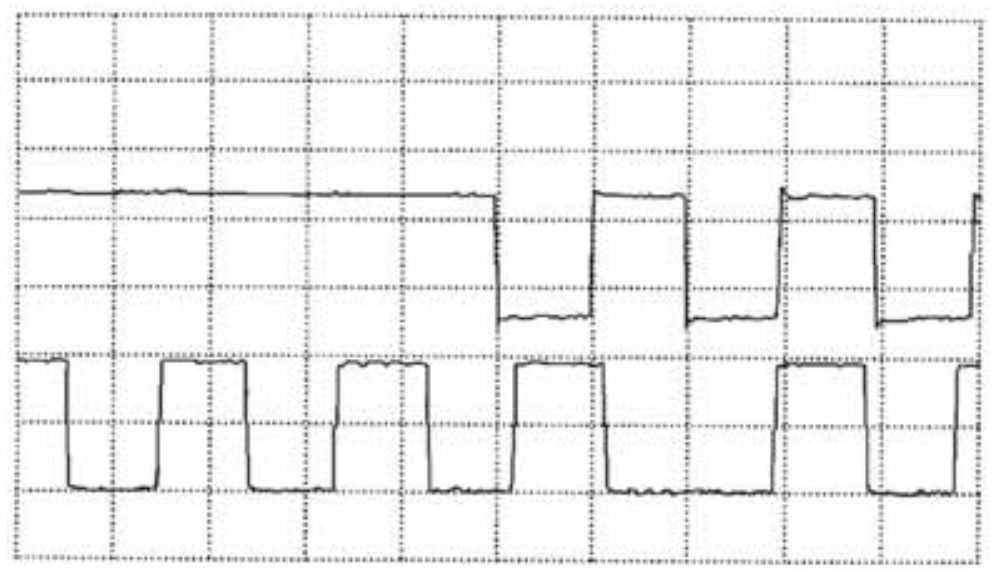

Figure 8. Effect of resetting the counter in the feedback loop. Vertical scale $=2 \mathrm{~V} /$ div; horizontal scale $=5 / \mathrm{sec} /$ div.

\section{CONCLUSIONS}

The monitoring instrument described is capable of recording long monitoring periods using a small amount of memory; since its construction is quite simple, its cost is low and is well suited to be installed in residential environments. Also, its operating principle does not involve an integrating procedure, as in RMS-value meters; thus, fast transients such as those produced by starting motors can be recorded. Special care was put into the recovery of the instrument to power outages, since quite often voltage fluctuations occur prior and after the interruption.

At its present configuration, with 32 samples per cycle, the amount of memory required to store the information occupies about a quarter of the memory map of a common 8-bit microprocessor. By doubling the amount of memory it can be easily upgraded to 64 samples per cycle. The information recorded can be used to identify and assess lowvoltage problems; and to better specify uninterruptible power supplies.

\section{REFERENCES}

[1] Reid W., "Power Quality Issues - Standards and Guidelines". IEEE Transactions on Industry Applications, Vol. 32, No. 3, May/June, 1996, pp. 625-632.

[2] IEEE Working Group on Voltage Flicker and Service to Critical Loads "Power Quality - Two Different Perspectives". IEEE Transactions on Power Delivery, Vol. 5, No. 3, July, 1990, pp. 1501-1513.

[3] Sutherland, P., "Harmonic Measurements in Industrial Power Systems", IEEE Transactions on Industry Applications, Vol. 31, No. 1, January/February, 1995, pp. 175-183.

[4] Simpson R., "Instrumentation, Measurement Techniques and Analytical Tools in Power Quality Studies", IEEE Transactions on Industry Applications, Vol. 34, No. 3, May/June, 1998, pp. 534-548.

[5] Martzloff F. and Grutz T., "Power Quality Site Surveys: Facts, Fiction and Fallacies". IEEE Industrial Applications Society Industrial and Commercial Power Conference Record, May, 1987, pp. 21-33.

[6] Perez S., Calva M. \& Castañeda R., "A Microcontroller-Based Data Logging System". Instrumentation \& Depelopment, Vol. 3, No. 8, 1997, pp. 24-30.

[7] Jou H., Chu H. \& Huang C., "New Method for Reducing the Transient Response Time of an AC Voltage Detector." International Journal of Electronics, Vol. 70, No. 2, 1991, pp. 367-375.

[8] Calleja H., "Dynamic Clamp for Pull-In Time Reduction". IEEE Transaction on Measurement and Instrumentation, Vol. 45, No. 5, October, 1996, pp. 907-909. 
Authors Byography

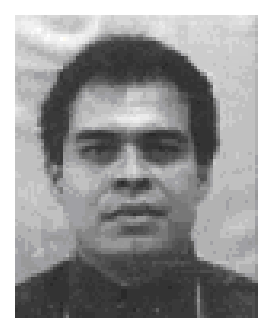

Manuel Gracida Aguirre

Was bom in México City in 1968. He received the B.Sc. degree from the Minatitlan Institute of Technology (ITM) in 1993, and the M.Sc. degree from the National Center for Research and Development of Technology, 1996. From 1996 to 2001 he worked at the ITM, in charge of projects in the field of electronic instrumentation for the oil industry. In 2001 he joint the Cerro Azul Institute of Technology, were he worked in the development of data adquisition boards for agricultural applications. He is currently working toward a Ph. D. degree at the University of Valencia.

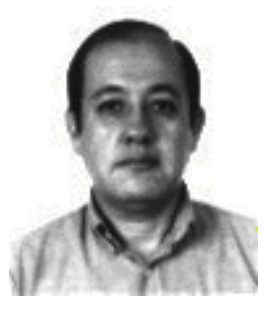

\section{Hugo Calleja}

Was born in Oaxaca, Mexico, in 1954. He received the B.Sc. degree from the National Polytechnic Institute (IPN), 1977, the M.Sc. degree from the National Autonomous University (UNAM), 1981, and the Ph.D. degree from the National Center for Research and Development of Technology (Cenidet), 2000, all in electrical engineering. He has worked at the Engineering Faculty, UNAM; and at the Institute for Electrical Research, in charge of the development of measuring equipment. Since 1993 he has been a professor at Cenidet, His research interests include electronic instrumentation for power electronics, and electric energy measuring. He is author of a book on electronic circuits for data acquisition. He received the Gabino Barreda Medal, granted by UNAM. He is a member of the SNI, and a Senior Member of the IEEE. 